\title{
INCIDÊNCIA DE INFECÇÃO DE SÍTIO CIRÚRGICO EM HOSPITAL DIA: COORTE DE 74.213 PACIENTES MONITORADOS
}

\author{
Incidence of infection of surgical site in hospital day: cohort of 74,213 patients monitored
}

Incidencia de la infección del sitio quirúrgico en el día de hospital: cohorte de 74,213 pacientes monitoreados

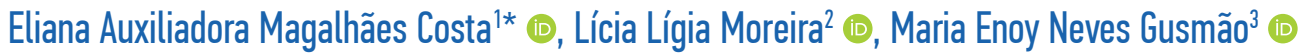

RESUMO: Objetivos: Descrever a incidência de infecção do sítio cirúrgico (ISC) em seguimento após alta em hospital dia (HD) e comparar esses indicadores com dados de hospitais convencionais. Método: Estudo de coorte histórica composto de 74.213 pacientes operados e monitorados num HD de Salvador (BA), entre 2012 e 2017. Resultados: No período estudado, o sistema de vigilância do HD monitorou 85,1\% dos pacientes após a alta e foi identificada incidência total de ISC de $0,3 \%$, com variação de 0,2 a 0,4\% entre os anos, taxas estatisticamente menores do que as reportadas para ISC em regime de internação hospitalar. Conclusão: Os indicadores de ISC revelados neste estudo ratificam que a modalidade da assistência cirúrgica ambulatorial porta menor risco de aquisição de infecção para os pacientes operados, quando comparados com os dados de infecção cirúrgica de pacientes em hospitais convencionais. Entretanto, torna-se indispensável um sistema de seguimento dos pacientes após a alta, no sentido de evitar a subnotificação e os sub-registros dos dados de ISC, pois na ausência de ambos se podem ocultar riscos e identificar taxas irreais. Palavras-chave: Infecção da ferida cirúrgica. Hospital dia. Segurança do paciente.

ABSTRACT: Objectives: To describe the incidence of surgical site infection (SSI) after discharge from a Daycare Unit (DU) and to compare these indicators with data from conventional hospitals. Method: This is a historical cohort study including 74,213 patients who underwent surgery and were monitored at a DU in Salvador (Bahia State, Brazil), between 2012 and 2017. Results: During the studied period, the DU surveillance system monitored 85.1\% of patients after discharge. We found a total SSI incidence of $0.3 \%$, varying between 0.2 and $0.4 \%$ in those years. These rates were statistically lower than those reported for SSI in hospitalized patients. Conclusion: The SSI indicators revealed in this study confirm that outpatient surgical care poses lower risks of infection acquisition for the operated patients, when compared with surgical infection data of patients from conventional hospitals. However, a follow-up system for patients after discharge is essential to avoid sub-reporting and sub-records regarding SSI data, considering that risks can be hidden and unrealistic rates can be identified in their absence. Keywords: Surgical wound infection. Day care, medical. Patient safety.

RESUMEN: Objetivos: Describir la incidencia de la infección del sitio quirúrgico (ISQ), después del alta del centro de día (CD) y comparar esos indicadores con los datos de hospitales convencionales. Método: Estudio de cohorte histórico con 74,213 pacientes operados y monitoreados en un CD en Salvador (Bahia, Brasil), entre 2012 y 2017. Resultados: Durante el período estudiado, el sistema de vigilancia del CD monitorizó el 85,1\% de los pacientes después del alta, y se identificó una incidencia total de la ISQ del 0,3\%, que varía desde el 0,2\% hasta el 0,4\% entre los años. Esas tasas son estadísticamente más bajas que las reportadas para ISQ bajo el régimen de hospitalización integral. Conclusión: Los indicadores de la ISQ revelados en este estudio confirman que la modalidad de atención quirúrgica ambulatoria conlleva un menor riesgo de adquisición de infección para los pacientes operados, en comparación con los datos de infección quirúrgica de pacientes en hospitales convencionales. Sin embargo, es indispensable un sistema de seguimiento para los pacientes después del alta hospitalaria, para evitar el sub-reporto y los subregistros de los datos de la ISQ, ya que en ausencia de ambos se pueden ocultar riesgos y se pueden identificar tasas poco realistas.

Palabras clave: Infección de la herida quirúrgica. Centro de atención diurna. Seguridad del paciente.

'Enfermeira; Graduada pela Universidade Estadual Paulista Paulista “Júlio de Mesquita Filho" (UNESP) - Botucatu (SP), Brasil.

${ }^{2}$ Mestre em Ciências da Saúde. Enfermeira de Controle de Infecção Hospitalar do Serviço Especializado em Controle de Infecção Hospitalar do Ministério da Saúde - Salvador (BA), Brasil.

${ }^{3}$ Doutora em Saúde Pública. Professora adjunta da Universidade Federal da Bahia (UFBA) - Salvador (BA), Brasil.

*Autor correspondente: costaeliana2003@hotmail.com

Recebido: 10/03/2019 - Aprovado: 16/06/2019

DOl: $10.5327 / 21414-4425201900040006$ 


\section{INTRODUÇÃO}

A despeito de todo o avanço do conhecimento técnico-científico e do incremento tecnológico incorporado pela área da saúde, as infecções relacionadas à assistência à saúde (IRAS) ainda constituem uma ameaça à segurança dos pacientes em todo o mundo.

Entre essas infecções, a do sítio cirúrgico (ISC) é considerada a mais comum do cuidado de saúde, acometendo $31 \%$ de todas as IRAS entre pacientes hospitalizados, associada com taxa de mortalidade de $3 \%$ e com $75 \%$ das mortes decorrentes de procedimentos cirúrgicos. Apesar desse potencial negativo, essas infecções podem ser evitadas em até $60 \%$ dos casos, por meio da adoção de medidas de prevenção e controle ${ }^{1-4}$.

Conceitualmente, ISC refere-se à infecção que ocorre após cirurgia na parte do corpo em que foi realizado o procedimento e pode envolver pele, tecidos, órgãos ou material implantado, nos primeiros 30 dias ou até 90 dias, se houver colocação de implantes ${ }^{1-3}$.

O desenvolvimento de uma ISC causa aumento substancial na carga clínica, psicológica e econômica da cirurgia, atribuída ao aumento dos custos diretos pelo prolongamento do tempo de internação do paciente, dos testes diagnósticos e tratamentos, onerando os sistemas de saúde, principalmente os públicos. Pacientes com ISC têm duas vezes mais chances de morrer ou de necessitar de um cuidado intensivo e cinco vezes mais chances de ser re-hospitalizado. Os custos de hospitalização são o dobro para um paciente com infecção, quando comparados com os de um paciente operado e sem infecção $0^{5,6}$.

Adicionalmente, as ISC impactam desfavoravelmente na saúde mental e física dos pacientes, que podem experienciar desde retardamento da cura da ferida até complicações secundárias. Istso eleva, consequentemente, sua morbimortalidade a outras situações intangíveis, como dor, angústia, ansiedade, afastamento prolongado da casa, da família e do trabalho ${ }^{5}$.

Muitos fatores contribuem para o risco dessas infecções, tais como os relacionados ao paciente e à equipe, mas, essencialmente, da possibilidade de contaminação da ferida cirúrgica durante o procedimento operatório, período trans-operatório e, portanto, quanto maior a contaminação microbiana nesse período, maior a chance de infecção por conta da introdução de microrganismos na cavidade cirúrgica aberta e manipulada ${ }^{6,7}$.
A vigilância epidemiológica da ISC é uma estratégia importante para a qualidade e segurança do paciente operado. Tal vigilância se dá-se por meio do monitoramento sistemático dos dados de infecção e da possibilidade da adoção de medidas de prevenção e controle desses agravos. Entretanto, a maioria desses sistemas de vigilância é direcionada para pacientes hospitalizados, e poucos acompanham os que já tiveram alta hospitalar. Isso contribui para a subnotificação dessas infecções, uma vez que de 12 a $84 \%$ das ISC são manifestadas após a alta, enfatizando, desse modo, a relevância de um sistema de vigilância após a altaa ${ }^{6,8,9}$.

Estudos da Organização Mundial da Saúde (OMS) mostram que as ISC acometem um terço dos pacientes operados, e sua incidência é variável entre países pobres e em desenvolvimento (11,8 por 100 cirurgias, variação de 1,2 a 23,6), com taxas em países europeus de 9,5\% (cirurgias de cólon), 3,5\% (cirurgias cardíacas), 2,9\% (cesarianas), 1,4\% (colecistectomias) e $1,0 \%$ (cirurgias ortopédicas) $^{2}$.

Uma revisão sistemática realizada por pesquisadores europeus em hospitais da França, Alemanha, Itália, Espanha e Reino Unido confirmou que há um número significativo de ISC ocorrendo em várias especialidades cirúrgicas na Europa: cirurgias cardiotorácicas (taxa de ISC de 9,2\% no Reino Unido, de 1,9 a 2,5\% na França), cirurgias gerais (taxa de $7,8 \%$ no Reino Unido) e cirurgias otorrinolaringológicas (taxa de $36 \%$ na França) $)^{5}$.

Pesquisa realizada em hospitais de 16 cidades da Turquia identificou taxa geral de ISC de 4,3\% entre 41.563 procedimentos. ${ }^{10}$ Estudo semelhante em seis cidades da Índia reportou taxa de $4,2 \%$ de ISC entre 28.340 cirurgias $^{11}$. Estudo internacional, que objetivou determinar o impacto da ISC nos custos dos cuidados de saúde em procedimentos ambulatoriais, por meio de uma coorte retrospectiva de pacientes, identificou taxas de $0,3 \%$ e $0,5 \%$ (colecistectomias), $0,6 \%$ e $0,5 \%$ (herniorrafias) e $0,8 \%$ (cirurgias de mama) ) $^{12.12} \mathrm{Na}$ Colômbia, foram observados 193 casos de ISC em 5.063 procedimentos realizados $(3,8 \%)^{13}$.

No Brasil, a avaliação pioneira da incidência de infecção cirúrgica data do ano 2000, num estudo prospectivo de 23 anos realizado no Recife, Pernambuco, que identificou incidência total de ISC de 11\% (1.622 casos em 14.694 cirurgias) e taxa de ISC em cirurgias limpas de 5,8\%. Esse estudo reporta taxa total de ISC em cirurgias ambulatoriais de $0,4 \%$ (130 casos em 27.580 procedimentos) e taxa de ISC em cirurgia limpa de $0,5 \%{ }^{14}$.

Estudo que investigou a ocorrência de ISC após alta em ambulatório de egressos em hospital universitário brasileiro identificou que, em 2.772 procedimentos realizados na 
especialidade de cirurgia geral, compareceram ao acompanhamento após alta 2.283 pacientes (82\%), com 85 casos de infecção e incidência total de $3,7 \%{ }^{6}$.

A despeito do crescente número de estudos publicados sobre as ISC, poucos dados, como os aqui citados, são publicados acerca da incidência dessas infecções em seguimento após a alta, especificamente em serviços de saúde não convencionais, como os hospitais dia (HD), constituindo uma lacuna importante do conhecimento, haja vista que a cirurgia no regime ambulatorial é uma tendência em ascensão que permite grandes benefícios, tais como menor custo, maior número de atendimento e, a priori, menor risco de infecção.

Nesse sentido, este estudo procura responder à seguinte questão central: qual a incidência da infecção do sítio cirúrgico em seguimento após alta e realizada em Hospital Dia? Essas infecções são comparativamente menores do que as realizadas em hospitais convencionais?

\section{OBJETIVO}

Tem por objetivo descrever a incidência de infecção do sítio cirúrgico em seguimento após alta em HD e comparar esses indicadores com hospitais convencionais.

\section{MÉTODO}

Trata-se de um estudo de coorte retrospectiva, desenho que permite a observação de grupos expostos (pacientes operados em HD) a um fator de risco suposto como causa de doença a ser detectada no futuro ${ }^{15}$. Utilizou-se o conceito de HD como "assistência intermediária entre a internação e o atendimento ambulatorial para a realização de procedimentos clínicos ou cirúrgicos, que requeiram a permanência do paciente na unidade por período máximo de 12 horas", definido em legislação.

O HD estudado é uma organização localizada em Salvador, Bahia, Brasil, de caráter privado, conveniado, que realiza exclusivamente procedimentos cirúrgicos em regime de HD, com movimento de aproximadamente 1.000 cirurgias/mês e média de 12 mil procedimentos cirúrgicos/ano. As cirurgias mais frequentemente realizadas nesse serviço e que constam deste estudo são cirurgias das especialidades: geral (hernioplastia, exérese de lesão de pele e de nódulo mamário), otorrinolaringologia (amigdalectomia, tonsilectomia), vascular (exérese de varizes), cirurgia de mão, plástica, histeroscopias, oftalmológica (facectomia), hemorroidectomia, entre outras.

Nesse hospital, o Serviço de Controle de Infecção Hospitalar $(\mathrm{SCIH})$ possui um sistema de vigilância epidemiológica para todo paciente operado na instituição, que consiste no seguimento do paciente durante a internação e após a alta. São excluídos desse sistema de vigilância, os pacientes admitidos para realização de exames endoscópicos e outros procedimentos considerados não cirúrgicos, a exemplo de implantação de cateter central para quimioterapia, laser e retirada de duplo J.

Cada paciente operado possui uma ficha de acompanhamento de IRAS, contendo os seguintes dados: identificação, idade, telefone, diagnóstico de base, data e tipo de cirurgia, nome e número do conselho regional do cirurgião, tempo cirúrgico, sistema de classificação clínica (ASA), uso de antibiótico profilático e intercorrências presentes durante $\mathrm{o}$ período do transoperatório.

O seguimento após a alta é realizado por três estagiárias de enfermagem do $\mathrm{SCIH}$, devidamente treinadas e sistematicamente supervisionadas e consiste em contatar, por telefone, todo paciente operado, dentro de até 28 a 30 dias decorridos da cirurgia, com o objetivo de identificar sua evolução no pós-operatório e possíveis eventos adversos, incluindo infecção após procedimento cirúrgico e que se manifestou após a alta da instituição. Nesse contato telefônico, as estagiárias seguem um procedimento padrão, na tentativa de identificar como está o estado geral do paciente, condições da incisão cirúrgica, presença de secreções ou febre, retorno à consulta médica e uso de medicações com sutileza, de modo a minimizar possíveis induções nas respostas. É padronizado o máximo de três tentativas telefônicas por paciente e, na impossibilidade do contato, ele é considerado "sem contato" e excluído do banco de dados de pacientes monitorados pelo SCIH. Os dados dos contatos telefônicos são registrados nas fichas dos pacientes.

Fizeram parte dessa coorte as fichas de acompanhamento de IRAS dos pacientes submetidos a procedimentos cirúrgicos no HD sede da pesquisa, no período compreendido entre os anos de 2012 e 2017, totalizando 74.213 pacientes monitorados, contatados no seguimento após a alta.

A coleta de dados foi realizada nos meses de janeiro e fevereiro de 2018, com o auxílio de um instrumento próprio para registro das variáveis de interesse desta pesquisa: número de cirurgias realizadas, número de pacientes monitorados após a alta pelo SCIH e número de ISC por potencial de contaminação das cirurgias. Foram utilizados os critérios diagnósticos de infecção hospitalar do sítio cirúrgico, adotados pela Agência Nacional de Vigilância Sanitária (ANVISA) ${ }^{3} .3$ 
Os dados foram armazenados e analisados no programa Epi Info.

O projeto de pesquisa foi submetido ao Comitê de Ética em Pesquisa e aprovado segundo Certificado de Apresentação para Apreciação Ética (CAAE) nº 84696018.9.0000.0057.

\section{RESULTADOS}

A distribuição dos procedimentos cirúrgicos realizados e monitorados no HD estudado encontra-se na Tabela 1.

Na Tabela 1, verifica-se que, entre 2012 e 2017, foram operados no hospital em estudo 87.166 pacientes. Destes, foram monitorados após a alta 73.734 pacientes. A proporção de pacientes monitorados entre os anos estudados variou de 78,7 (2016) a 90,6\% (2012), com total de $85,1 \%$ de pacientes acompanhados após a alta hospitalar.

A Tabela 2 apresenta a incidência de ISC em pacientes monitorados no HD, segundo os anos de estudo. Observa-se incidência total de ISC de $0,3 \%$ e incidência anual quase constante durante o período analisado, com variação de $0,2 \%$ em 2013; 0,3\% em 2012 e 2015; e 0,4\% em 2014 e 2017.

A incidência de ISC segundo o potencial de contaminação das cirurgias é apresentado na Tabela acima. Observa-se durante o período estudado, 177 infecções do sítio cirúrgico $(0,4 \%)$ entre as 41.771 cirurgias classificadas como limpas, 15 infecções entre as cirurgias potencialmente contaminadas (0,2\%), 39 ISC nas cirurgias contaminadas (0,2\%) e nenhuma infecção em cirurgia classificada como infectada. Os dados da Tabela 3 também demonstram que entre as 73.734 cirurgias monitoradas nos anos estudadas, 56,6\% foram cirurgias limpas (41.771/ 73.734), 32,1\% foram cirurgias contaminadas

Tabela 1. Distribuição proporcional de pacientes monitorados entre os procedimentos cirúrgicos realizados, segundo o ano, no hospital dia.

\begin{tabular}{|l|c|c|c|}
\multirow{2}{*}{ Período } & $\begin{array}{c}\text { Cirurgias } \\
\text { realizadas }\end{array}$ & \multicolumn{2}{|c|}{$\begin{array}{c}\text { Número de } \\
\text { pacientes monitorados }\end{array}$} \\
\cline { 3 - 4 } & Número & $\%$ \\
\hline 2012 & 12.769 & 11.577 & 90,6 \\
\hline 2013 & 13.557 & 12.002 & 88,5 \\
\hline 2014 & 13.271 & 11.292 & 85,1 \\
\hline 2015 & 14.710 & 12.723 & 86,5 \\
\hline 2016 & 16.211 & 12.754 & 78,7 \\
\hline 2017 & 16.648 & 13.865 & 83,3 \\
\hline Total & 87.166 & 74.213 & 85,1 \\
\hline
\end{tabular}

(23.701/73.734), 11,8\% foram cirurgias potencialmente contaminadas $(8.724 / 73.734)$ e apenas $0,02 \%$ de cirurgias infectadas $(17 / 73.734)$.

\section{DISCUSSÃO}

Neste estudo, identificou-se que o sistema de vigilância após a alta dos pacientes operados, implantado no HD sede da pesquisa monitorou o percentual total de $85,1 \%$ da coorte de 73.734 pacientes. Tal monitoramento foi considerado significativo e em sintonia com um estudo em um hospital universitário nacional ${ }^{6}$ no qual os autores acompanharam, após a alta, 82\% dos pacientes em ambulatório de egressos cirúrgicos, validando a metodologia de contatos telefônicos. Esta, quando bem sistematizada, constitui um método efetivo de monitoramento de pacientes cirúrgicos pós-alta, como aqui verificado.

Entre os anos de 2012 e 2017, identificou-se incidência total de ISC no HD de $0,3 \%$, com variação entre 0,2 e $0,4 \%$, taxas inferiores às reportadas para ISC em hospitais convencionais internacionais, a exemplo dos percentuais citados pela $\mathrm{OMS}^{2}(11,8 \%)$, das taxas em hospitais convencionais de países europeus 5 (7,8; 8,6 e 3,2\%), taxas de hospitais da Turquia e Índia ${ }^{10,11}\left(4,3\right.$ e 4,2\%) e da Colômbia $(3,8 \%)^{13}$.

Ainda comparando as taxas de ISC do presente estudo com duas pesquisas nacionais realizadas em hospitais convencionais $^{6,15}$, obtiveram-se taxas bem menores no HD, ratificando a cirurgia em regime ambulatorial como de menor risco de infecção.

A incidência total de $0,3 \%$ de ISC apontada neste estudo se assemelha aos dados de um estudo internacional em

Tabela 2. Incidência de infecção do sítio cirúrgico em pacientes monitorados, segundo o ano de estudo, no hospital dia.

\begin{tabular}{|l|c|c|c|}
\multirow{2}{*}{ Período } & Número de & \multicolumn{2}{|c|}{$\begin{array}{c}\text { Infecção do } \\
\text { Sacientes } \\
\text { monitorados Cirúrgico }\end{array}$} \\
\cline { 3 - 4 } & 11.577 & 32 & $\%$ \\
\hline 2012 & 12.002 & 28 & 0,3 \\
\hline 2013 & 11.292 & 41 & 0,2 \\
\hline 2014 & 12.723 & 43 & 0,4 \\
\hline 2015 & 12.275 & 36 & 0,3 \\
\hline 2016 & 13.865 & 51 & 0,3 \\
\hline 2017 & 73.734 & 231 & 0,4 \\
\hline Total & & & 0,3 \\
\hline
\end{tabular}


Tabela 3. Incidência de infecção do sítio cirúrgico (ISC) nas cirurgias monitoradas, segundo o potencial de contaminação no hospital dia.

\begin{tabular}{|c|c|c|c|c|}
\hline $\begin{array}{l}\text { Período } \\
\text { (ano) }\end{array}$ & $\begin{array}{c}\text { Cirurgias limpas } \\
\mathbf{N} \\
(\mathbf{n} / \mathrm{ISC} \%)\end{array}$ & $\begin{array}{c}\text { Cirurgias } \\
\text { potencialmente } \\
\text { contaminadas } \\
\text { N } \\
(\mathrm{n} / \mathrm{ISC} \%)\end{array}$ & $\begin{array}{c}\text { Cirurgias } \\
\text { contaminadas } \\
N \\
(\mathrm{n} / \mathrm{ISC} \%)\end{array}$ & $\begin{array}{c}\text { Cirurgias infectadas } \\
\mathbf{N} \\
(\mathrm{n} / \mathrm{ISC} \%)\end{array}$ \\
\hline 2012 & $\begin{array}{c}7.052 \\
(26 / 0,4 \%)\end{array}$ & $\begin{array}{c}1.778 \\
(3 / 0,2 \%)\end{array}$ & $\begin{array}{c}2.742 \\
(3 / 0,1 \%)\end{array}$ & $\begin{array}{c}05 \\
-\end{array}$ \\
\hline 2013 & $\begin{array}{c}6.624 \\
(19 / 0,3 \%)\end{array}$ & $\begin{array}{c}2.175 \\
(4 / 0,2 \%)\end{array}$ & $\begin{array}{c}3.201 \\
(5 / 0,2 \%)\end{array}$ & $\begin{array}{c}02 \\
-\end{array}$ \\
\hline 2014 & $\begin{array}{c}6.338 \\
(33 / 0,5 \%)\end{array}$ & $\begin{array}{c}1.191 \\
(1 / 0,08 \%)\end{array}$ & $\begin{array}{c}3.761 \\
(7 / 0,2 \%)\end{array}$ & $\begin{array}{c}02 \\
-\end{array}$ \\
\hline 2015 & $\begin{array}{c}7.038 \\
(30 / 0,4 \%)\end{array}$ & $\begin{array}{c}1.314 \\
(2 / 0,1 \%)\end{array}$ & $\begin{array}{c}4.371 \\
(11 / 0,2 \%)\end{array}$ & - \\
\hline 2016 & $\begin{array}{c}7.169 \\
(29 / 0,4 \%)\end{array}$ & $\begin{array}{c}976 \\
0\end{array}$ & $\begin{array}{c}4.608 \\
(07 / 1,8 \%)\end{array}$ & $\begin{array}{l}01 \\
-\end{array}$ \\
\hline 2017 & $\begin{array}{c}7.550 \\
(40 / 0,5 \%)\end{array}$ & $\begin{array}{c}1.290 \\
(5 / 0,4 \%)\end{array}$ & $\begin{array}{c}5.018 \\
(06 / 0,1 \%)\end{array}$ & $\begin{array}{l}07 \\
-\end{array}$ \\
\hline Total & $\begin{array}{c}41.771 \\
(177 / 0,4 \%)\end{array}$ & $\begin{array}{c}8.724 \\
(15 / 0,2 \%)\end{array}$ & $\begin{array}{c}23.701 \\
(39 / 0,2 \%)\end{array}$ & $\begin{array}{l}17 \\
(0)\end{array}$ \\
\hline
\end{tabular}

procedimentos cirúrgicos ambulatoriais ${ }^{12}$ que também utilizou uma coorte retrospectiva de pacientes e identificou taxas de 0,3 e $0,5 \%$ (colecistectomias), 0,5 e $0,6 \%$ (herniorrafias) e $0,8 \%$ (cirurgias de mama), bem como aos dados reportados num hospital universitário do Recife ${ }^{14}$, que identificou $0,4 \%$ de infecção cirúrgica no ambulatório de egressos.

A taxa de infecção em cirurgia limpa é um indicador da qualidade cirúrgica da instituição e a literatura preconiza, sem especificar para que tipo de serviço cirúrgico, se convencional ou em regime ambulatorial, que essas taxas de infecção devem ser menores do que $2 \%$. Para as cirurgias classificadas como potencialmente contaminadas, contaminadas e infectadas, taxas menores do que $10 \%$, taxas de $20 \%$ e de 30 a $40 \%$, respectivamente, são as aceitáveis ${ }^{15}$.

No HD estudado, identificou-se taxa de ISC em cirurgias limpas de $0,4 \%$ e de $0,2 \%$ em cirurgias potencialmente contaminadas e contaminadas, percentuais significativamente menores do que os recomendados pela literatura e em alguns estudos internacionais $(3,5 ; 2,5 ; 6,8 \text { e } 8,6 \%)^{4,9}$.

O percentual total de $0,3 \%$ de ISC ao longo dos anos estudados nesse HD e o percentual total de $0,4 \%$ para ISC em cirurgias limpas revelam não apenas a excelência cirúrgica desse serviço, mas que essas taxas são bem menores do que as reportadas em pacientes que operam em serviços cirúrgicos convencionais, ratificando que a modalidade da assistência ambulatorial porta menor risco de aquisição de infecção para os pacientes operados, quando comparados com os dados de cirurgias de pacientes em regime de hospitais convencionais.

Entretanto, torna-se indispensável um sistema de seguimento dos pacientes após a alta, no sentido de evitar a subnotificação e os sub-registros dos dados, pois na ausência destesde ambos se podem ocultar informações e identificar taxas irreais.

\section{CONCLUSÃO}

Este estudo alcançou seu objetivo e respondeu às perguntas norteadoras, na medida em que identificou a incidência de ISC em seguimento de pacientes após a alta hospitalar (incidência total de $0,3 \%$, entre os anos 2012 e 2017) e comparou esses indicadores com os dados de hospitais convencionais.

Os resultados sinalizaram que a adequação do sistema de vigilância epidemiológica das infecções cirúrgicas adotado no HD sede da pesquisa se mostrou robusto ao possibilitar acompanhamento total de $85,1 \%$ dos pacientes após alta, bem como a elaboração de um banco de dados de indicadores de infecção cirúrgica da instituição.

Os percentuais de ISC identificados no HD estudado ratificam a modalidade de cirurgia ambulatorial como de menor risco de ISC quando comparados com os dados de cirurgias realizadas em hospitais convencionais. 


\section{REFERÊNCIAS}

1. Center for Disease Control and Prevention. National Healthcare Safety Network. NHSN Patient Safety Component Manual. Surgical Site Infection Event. [acessado em 05.02.2018]. Disponível em: https:www.cdc.gov/nhsn/pdfs/validation/2017/pcsmanual_2017. pdf. Acesso em 05.02.2018.

2. World Health Organization. Global Guidelines for the Prevention of Surgical Site Infection. Geneva: OMS; 2016.

3. Agência Nacional de Vigilância Sanitária. Critérios diagnósticos de infecção relacionada à assistência à saúde. $2^{\mathrm{a}}$ ed. Brasília: ANVISA; 2017. v. 2.

4. Araújo IS, Carvalho R. Eventos graves em pacientes cirúrgicos: ocorrência e desfecho. Rev SOBECC. 2018;23(2):77-83. https://doi. org/10.5327/Z1414-4425201800020004

5. Badia JM, Casey AL, Petrosillo N, Hudson PM, Mitchell AS, Crosby C. Impact of surgical site infection on healthcare costs and patient outcomes: a systematic review in six European countries. J Hosp Infect. 2017;96(1):1-15. https://doi.org/10.1016/j.jhin.2017.03.004

6. Reis RG, Rodrigues MCS. Infecção do sítio cirúrgico após-alta: ocorrência e caracterização de egressos de cirurgia geral. Cogitare Enferm. 2017;22(4):e51678. http://dx.doi.org/10.5380/ce.v22i4.51678

7. Oliveira AC, Gama CS. Avaliação da adesão às medidas para a prevenção de infecções do sítio cirúrgico pela equipe cirúrgica. Rev Esc Enferm USP. 2015;49(5):767-74. https://doi.org/10.1590/ S0080-623420150000500009

8. Le Meur N, Grammatico-Guillon L, Wang S, Astagneau P. Health insurance database for post-discharge surveillance of surgical site infection following athroplasty. J Hosp Infect. 2016;92(2):140-6. http://dx.doi.org/10.1016/j.jhin.2015.10.006
9. Organização Mundial da Saúde. Segundo desafio global para a segurança do paciente: cirurgias seguras salvam vidas. Rio de Janeiro: Organização Pan-Americana da Saúde, Ministério da Saúde, Agência Nacional de Vigilância Sanitária; 2009.

10. Leblebicioglu H, Erben N, Rosenthal VD, Sener A, Uzun C, Send G, et al. Surgical site infection rates in 16 cities in Turkey: findings of the International Nosocomial Infection Control Consortium. Am J Infect Control. 2015;43(1):48-52. https://doi.org/10.1016/j.ajic.2014.09.017

11. Singh S, Chakravarthy M, Rosenthal VD, Myatra SN, Dwivedy A, Bagasrawala I, et al. Surgical site infection rates in six cities of India: findings of the International Nosocomial Infection Control Consortium. Am Int Health. 2015;7(5):354-9. https://doi.org/10.1093/inthealth/ihu089

12. Olsen MA, Tian F, Wallace AE, Nickel KB, Warren DK, Fraser VJ, et al. Use of quantile regression to determine the impacto $n$ total health care costs of surgical site infections following common ambulatory procedures. Ann Surg. 2016;12. http://dx.doi.org/10.1097/ SLA.0000000000001590

13. Motta NH, Bohrer CD, de Oliveira FG, Matos A, Alves DCI. Prevenção da infecção de sítio cirúrgico em hospital universitário: avaliação por indicadores. Vigil Sanit Debate. 2017;5(3):92-9. http://dx.doi. org/10.22239/2317-269x.00973

14. Ferraz EM, Ferraz AAB, Bacelar TS, D'Albuquerque HS, Vasconcelos MDM, Leão CS. Controle de infecção em cirurgia geral: resultado de um estudo prospectivo de 23 anos e 42.274 cirurgias. Rev Col Bras Cirurgiões. 2001;28(1). http://dx.doi.org/10.1590/ S0100-69912001000100005

15. Almeida Filho N, Barreto M. Desenhos de pesquisa epidemiológica. In: Almeida Filho N, Barreto M, editores. Epidemiologia \& saúde: fundamentos, métodos, aplicações. Rio de Janeiro: Guanabara Koogan; 2011. 